\title{
Codonopsis on Exercise Stress Ulcer rat stomach tissues and motilin content on HSP70 expression Hui-feng JING ${ }^{1, a}$
}

\author{
College of Physical Education,Yan'an University,Yan'an 716000,China \\ a Jinghuifeng112@126.com
}

Keywords: Codonopsis; Exercise stress ulcer; Heat shock protein 70

\begin{abstract}
Objective: To investigate the occurrence of exercise stress gastric ulcer during motilin hormone content and expression of HSP70 and dangshen on Exercise Stress Ulcer Prevention effect and its mechanism. Methods: 30 adult SD rats were divided into three groups, each 10, divided into the control group, movement control, movement dosing group, the establishment of Exercise Stress Gastric Ulcer Model. Each group of rats gastric mucosa pathological damage index scoring by immunohistochemical staining gastric mucosa HSP70 expression; gastric tissue content preparation, testing motilin. Results: Compared with quiet group, gastric motility group organization motilin levels were significantly increased, slightly increased HSP70 expression; compared with motion control, dosing rats gastric tissue motilin levels were significantly decline, HSP70 expression increased further. Conclusion: Codonopsis can prevent exercise stress gastric ulcer, the protective mechanism may reduce gastric tissue in rats motilin content and increase the expression of HSP70 related.

All organisms under stress conditions can be synthesized in a highly conserved protective protein that is HSPs, especially high-intensity sports exhaustive exercise as a source of stress, causing physiological functions of various organ systems of the human body changes over the past decade, people express when the body motion stress HSPs conducted experimental studies, have found that the expression of HSPs[1] in the brain, liver, heart, skeletal muscle, spleen and blood cells and other parts of the body after exercise , HSP70 family is the most important one of its kind HSP protect the gastric mucosa, but there are no studies on exercise and stomach tissues of HSP70 relations. Numerous studies show that high intensity exhaustive exercise, especially as a strong source of stress, the blood flow to reduce the gastrointestinal tract, increased production of free radicals, lipid peroxidation increased production, will eventually cause gastric mucosal protective effect and injury of loss of balance, causing Exercise Stress Ulcer[2]. Clinical studies have shown dangshen, spleen, stomach, regulate gastrointestinal function, antioxidant and immunomodulatory effects[3]. Expression and motilin content in this experiment study the role of HSP70 in gastric stress ulcer in sport and in prevention of Codonopsis.
\end{abstract}

\section{Materials and Methods}

1.1 Animals

SD adult male rats, weighing 240-300g, Xi'an Jiaotong University Laboratory Animal Center.

1.2 Reagents and Instruments

Codonopsis purchased from Shaanxi Institute of Chinese Medicine; Motilin radioimmunoassay kit (purchased from the East Asian Institute of Technology, People's Liberation Army General Hospital immunity).

1.3 Movement to establish stress ulcer model

Zhao Jingguo study combining exercise stress ulcer model[3]and[4]Bedford motion model, and ethology performance sports model as the basis for the establishment of specific training programs are shown in Table.

Movement model: Full training for six weeks, four weeks of basic training before the first five weeks into the high-intensity training (exercise intensity according to Bedford[12]rat body weight / VO rat model of incremental exercise load training regression equations established be determined). 
The first six weeks of the first five weeks of training load is substantially the same, but differ when the last training, according to the following exhaustive exercise program: The $35 \mathrm{~m} / \mathrm{min}$ running speed, until the animal can not keep rats at a predetermined speed, rats buttocks pressure in the cage wall, hind legs dragging the belt with the rotation after 30 seconds, when the stimulus driven brush invalid, reduce exercise load (by the VI $\rightarrow$ grade I load), continues to move to when the load level I sport animals failed adhere to the same level load running speed, has retained more than 3 times from $2 / 3$ end of the runway, signs failure, stimulate expulsion invalid ${ }^{[13]}$, the criteria for judging exhaustion.

Table 1 List of Laboratory Animal Movement programme

\begin{tabular}{ccccl}
\hline Week & speed $(\mathrm{m} / \mathrm{min})$ & Time $(\mathrm{min} / \mathrm{d})$ & Distance $(\mathrm{m})$ & Load Level \\
\hline 1 & 15 & 25 & 375 & Grade I load \\
2 & 20 & 30 & 600 & Grade II load \\
3 & 25 & 35 & 875 & Grade III load \\
4 & 30 & 40 & 1200 & Grade IV load \\
5 & 35 & 45 & 1575 & Grade V load \\
6 & 35 & 45 & 1575 & Grade VI load \\
\hline
\end{tabular}

1.4 Animal grouping

Experimental animals were randomly divided into three groups: sedentary group, without applying any sport, normal saline $(1 \mathrm{ml} / 100 \mathrm{~g})$; motion control group, normal saline $(1 \mathrm{ml} / 100 \mathrm{~g})$; sports dosing group, dangshen aqueous extract orally $(1 \mathrm{ml} / 100 \mathrm{~g})$.

1.5 Preparation and administration methods Codonopsis decoction

Press Codonopsis decoction of preparation[5] weighed 100g codonopsis, made the equivalent of $0.25 \mathrm{~g} / \mathrm{ml}$ of Codonopsis extract water, and stored at low temperatures set, after more than $3 \mathrm{~d}$ longer in use, re-extract. $25 \%$ Codonopsis decoction equivalent to $0.25 \mathrm{~g} / \mathrm{ml}$ crude drug. Each $2 \mathrm{ml}$ volume Codonopsis decoction gavage dosing group movement, quiet group and exercise control group in the same way to the appropriate amount of saline. Exercise group were in the same volume of saline or drenching after $2 \mathrm{~h}$ training.

\subsection{Test Method}

\subsubsection{Specimen collection}

Decapitated immediately remove the stomach in an ice tray, weighed filter paper to draw blood; then along the greater curvature of the stomach cut open flat, counts pathological injury index, while rapidly taking place in the antrum and lesions around $1 \mathrm{~mm} 2$ gastric placed into $10 \%$ formalin $\left(\mathrm{pH}\right.$ of $7.4,4{ }^{\circ} \mathrm{C}$ ) fixed after $48 \mathrm{~h}$, for pathology and immunohistochemistry. The remaining $10 \%$ Proventriculus weighing prepared homogenates centrifuged $\left(3000 \mathrm{rpm}, 10 \mathrm{~min}, 4{ }^{\circ} \mathrm{C}\right)$, the supernatant was stored at $1-4{ }^{\circ} \mathrm{C}$ refrigerator tested motilin content.

1.6.2 Determination ulcer index

Determination of gastric mucosal damage index reference Guth standard [6], calculated the percent inhibition of ulcers, ulcer inhibition rate $(\%)=($ total length of ulcers in the control group total length of ulcer treatment group) / total length of ulcers in the control group $\times 100 \%$.

1.6.3 Determination of the gastric mucosa motilin content

Motilin radioimmunoassay kits, according to the kit requirements to operate.

1.6.4 HSP70 expression was measured within the gastric mucosa

Using peroxidase labeled streptavidin method (SP) staining, and press kit requires immunohistochemistry operations. Light microscope, each slice randomly selected 10 high power field, using an image analysis software to measure the mean optical density value of immunohistochemical staining.

1.7 statistical processing

All the data is processed using SPSS13.0 software. The results were expressed as mean \pm standard deviation $( \pm \mathrm{s})$, $\mathrm{t}$ - test data, significant differences select $\mathrm{P}<0.05$ and $\mathrm{P}<0.01$ level. 


\section{Results}

2.1 Ulcer index in each group and ulcer inhibition rate rats

Table 2 Rats in each group for gastric ulcer and ulcer inhibition rate $(\mathrm{x} \pm \mathrm{s})$

\begin{tabular}{cccc}
\hline Groups & Number & Ulcer index & Ulcer inhibition rate \\
\hline NG & 10 & 0 & - \\
EG & 10 & $9.01 \pm 3.20^{\star \star}$ & - \\
EMG & 10 & $1.60 \pm 0.56^{\star \star}$ & 42.9
\end{tabular}

Note:NG Normal group;EG Exercise group;EMG Exercise and medicine group, $\mathbf{\Delta} \boldsymbol{\Delta}$ Represents a very quiet group has significant difference; $\star \star$ Represent motion control group with a very significant difference;

As can be seen from Table2, the ulcer index movement in rats dosing group slightly higher than sedentary control group was significantly lower than the control group movement $(\mathrm{P}<0.01)$.

2.2 Gastric tissues of rats motilin Contents

Table 3 The stomach in rats changes of the content of MOT $(x \pm s)$

\begin{tabular}{ccc}
\hline Groups & Number & Motilin(pg/ml) \\
\hline NG & 10 & $121.6 \pm 15.42$ \\
EG & 10 & $135.3 \pm 12.48^{\mathbf{\Delta}}$ \\
EMG & 10 & $122.8 \pm 6.24^{\star \star}$
\end{tabular}

Note: $\overline{N G ~ N o r m a l ~ g r o u p ; E G ~ E x e r c i s e ~ g r o u p ; E M G ~ E x e r c i s e ~ a n d ~ m e d i c i n e ~ g r o u p, ~} \mathbf{\Delta} \boldsymbol{\Delta}$ Represents a very quiet group has significant difference; $\star \star$ Represent motion control group with a very significant difference;

Table 3 shows that compared with the quiet group, the control group was significantly higher movement motilin content $(\mathrm{P}<0.01)$; compared with the motion control, dosing group motilin was significantly decreased $(\mathrm{P}<0.05)$; plus differences between drug group and the quiet group was not significant.

2.3 HSP70 immunohistochemical staining

Table 4 Rats stomach HSP70 positive cells compared menn optical density $(\mathrm{x} \pm \mathrm{s})$

\begin{tabular}{ccc}
\hline Groups & Number & $\begin{array}{c}\text { Average optical } \\
\text { density(meanOD) }\end{array}$ \\
\hline NG & 10 & $15.98 \pm 31.69$ \\
EG & 10 & $24.90 \pm 43.77^{\mathbf{}}$ \\
EMG & 10 & $52.34 \pm 41.65^{\mathbf{\Delta} \star \star}$
\end{tabular}

Note:NG Normal group;EG Exercise group;EMG Exercise and medicine group, $\boldsymbol{\Delta}$ Represents the quiet group than there are significant differences $\boldsymbol{\Delta} \boldsymbol{\Delta}$ Represents a very quiet group has significant difference; $\star \star$ Represent motion control group with a very significant difference;

\section{Analysis and discussion}

Codonopsis terpenes, for the treatment of acute and chronic gastritis and peptic ulcer, etc. Can significantly relieve inflammation mucosa, improving the quality of ulcer healing, prevention of gastric mucosal damage under stress. HSP is a class of highly conserved proteins, including two proteins, structural and inducible HSP70 HSP70[7]. Structural nascent protein HSP70 help form the correct structure and positioning, maintenance of cell protein homeostasis; and when the stress induced HSP70 proteins repair damaged, remove relevant, it can improve cell tolerance to ischemia and hypoxia and heat of[8]. Gastric major structural synthesis under normal conditions HSP70, to reduce the mucosal injury caused by repetitive stimulation of food, when stimulated by stress, stomach tissue induced HSP70 expression increased[9]. Previous studies showed that normal rat gastric glandular cells in the presence of HSP70, in order to express the extracellular paddle 
based[10]. Tsukimi et al[11] found that, HSP70 in normal gastric mucosa and gastric ulcer tissues were expressed, and the HSP70 transcripts ulcer tissue was higher than normal gastric mucosa, and HSP70 expression was significantly higher in ulcer formation process, HSP70 expression with the healing of ulcers and gradually increased, and gastric mucosal injury was a negative correlation. In case of gastric ulcer, the ulcer index will change, and the size of the ulcer and gastric mucosal injury index showing positive change relations, ulcer inhibition rate is an indicator of the improvement reflects gastric mucosal injury after dosing, the indicator is simple and effective.

The study found that gastric ulcer index of rats in the control group than the sport movement higher dosing group, the gastric mucosa HSP70 expression representing the movement of low dosing group, motilin peptide levels increased significantly, gastric mucosal injury is more serious. Because there may be: (1) large number of acidic substances, when in motion, precipitating a large number of apoptotic cells in the gastric mucosa, can be used for the synthesis of HSP70 gland cells decreased, HSP70 downregulation; (2) heat shock transcription factor (HSF) is the process of HSP70 expression an important regulatory factor, which can weaken the HSF1 activity, inhibition of HSP70 expression HSP70mRAN and, thus exacerbating the degree of ulcers; this experiment, rats under high-intensity exercise in the state, intake and poor absorption of nutrients, manifested as loss poor, slow weight gain, hair yellow, gloss differential, protein malnutrition, thereby inhibiting the expression of HSP70 synthesis and HSP70mRNA; induce the synthesis of HSP70 (3) exercise stress caused by movement of the timing of the body may be related to changes in the environment.

This study found that treatment can significantly reduce gastric Codonopsis ulcer index, gastric relatively elevated HSP70 expression, motilin peptide levels were significantly increased, compared alleviate gastric mucosal injury. It may be due to: Codonopsis adjustable gastric tissue motilin content, due to the inhibition of gastric ulcer symptoms of hyperthyroidism caused by movement; Codonopsis inhibits exercise-induced increase in glandular tissue acetylcholine content, reducing acetylcholine and motilin synergy the role of the glandular tissue of the stomach contents moving maintained at a relatively stable level, to improve the gastric mucosal blood flow, and thus play a very good protective effect of gastric mucosa; Codonopsis induced HSP70 expression in order to improve the ischemic tolerance of two aspects, injury may have enhanced anti-stress role of protection mechanisms; Codonopsis by inducing HSP70 expression, inhibit the production of oxygen free radicals key enzyme oxidase, the feedback effect of reducing the generation of oxygen free radicals;Rokutan et al found that HSP70 as a chaperone, to protect the mitochondria and cell apoptosis, reduce internal stress-induced cell protein denaturation and aggregation. Codonopsis by inducing HSP70 expression, in the stress of restoring and maintaining the activity of antioxidant enzymes, while repairing damaged when the stress function of the protein structure, and thus play a protective role in cells. Pathway leading to tissue ischemia and hypoxia cell damage more recent studies have shown that over-expression of HSP70 in the stomach by increasing the gastric mucosal blood flow to play a protective role in the cells, which play a role in anti-ulcer, and after sympathectomy research was found to reduce gastric mucosal blood flow in rats, can promote the formation of stomach ulcers, which may be inferred HSP70 play a protective role by increasing the gastric mucosal blood flow; HSP70 may also by promoting intracellular gluconeogenesis and glycogen generation role, so intracellular glycogen storage increased, thereby enhancing the ability of stress.

Heat shock protein as an indicator of cellular stress activity of different forms of exercise intensity, its reaction and adaptation are very different, although overseas to explore the effects of different sports aspects of heat shock proteins has been a lot of research, but the domestic About movement and heat shock protein has just started, and mostly to explore different intensity, different periods of motion shock protein expression in skeletal and cardiac muscle heat, study for gastric tissue during exercise stress heat shock proteins is a blank. 


\section{Summary}

Codonopsis cure gastric ulcer induced by exercise stress induced HSP70 and its close relationship with a lot of expression, which is for the treatment of exercise-induced stress ulcer provides a new way of thinking. However, the expression of HSP70 is affected by many factors, the relationship between HSP70 and exercise stress gastric ulcer also remains to be further studied, but the positive is that, if we can divide the use of HSP70, will play a unique advantage in the field of sports medicine.

\section{Reference}

[1] Hernando R,Manso R.Muscle fibre stress in response to exercise:synthesis,Accumulation and isoform transitions of 70-k Daheat-shock proteins,J.EurJBiochem,1997,(243):460-467.

[2] Lowenstein $\mathrm{DH}$,Chan $\mathrm{Ph}$,Mile MT.The stress protein response in cultures neurons: Characterization and evidence for aprotectiverolein excitotoxicity,J.Neuron,1999,(7):1053.

[3] Fehrenbach E,Niess AM,Schlotz E,etal.Transcriptional and translation regulation of heat shock protein sinleukocytes of endurancerunners,J.ApplPhysiol,2000,(89):704-710.

[4] Bedford TG,Tipton CM,Wilson CN.Maximal oxygen consumption of rats and its changes with various experimental procedures,J.Appl Physiol,1979,(47):1278-1283.

[5] Guth, PH, et al. Topical aspirin plus $\mathrm{Hcl}$ gastric lesion in the rat cytoprotective effect of prosta glandin cimctidine and probanthine,J. Gastroenterlogy, 1987, 57(6):85-87.

[6] Wischmeyer PE,etal.Glutamine Induces Heat Shock Protein and Protects against Endotoxin Shock in the Rat,J.App Physiol,2001,90(6):2403-2410.

[7] Sato S,Abe K,Kawago EJ,etal.Isolation of Comple-ment Ary DNAs for Heat ShockProtein (HSP)70 and Heat Shock Cognate Protein(HSC)70 Genes and the expressions in Post isChaemic GerbilBrain,J.NeurolRes, 1992,14(5):375-380.

[8] Malago JJ,Koninkx JF,Vandijk JE.The heat shock response and cytoprotection of the intestinal epithelium,J.Cell Stress Chaperones,2002,7:191-199.

[9] Tsukimi Y,Nakai H,Itoh S,Amagase K,Okabe S.1nvolvement of heat shock proteins in the healing of acetic acid-induced gastric ulcers in rats,J. Physiol Pharmacol,2001, 52: 391-406.

[10] Kawai T, Teshima S, Knsumoto K, et al. A non-toxic heat shock protein 70 inducer, geranyl-geranylacetone, restores the heat shock response in gastric mucosa of pmtein malnourished rats,J.Lob Clip Med,2000,136(2):138.

[11] Kukreja Y,et al.Oxidant stress increases hsp70 mRNA in isolated perfused rat heart,J.Physiol ,267(heart circ physiol 36),1994,H2213-2219. 